\title{
Diabetic macular oedema: the effect of photocoagulation on fluorescein transport across the blood-retinal barrier
}

\author{
B Sander, M Larsen, C Engler, B Moldow, H Lund-Andersen
}

Br J Ophthalmol 2002;86: 1 139-1 142

See end of article for authors' affiliations

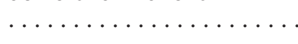

Correspondence to: Birgit Sander, Department of Ophthalmology, Herlev Hospital, University of Copenhagen, Dk 2730 Herlev, Denmark: bsander@idea.dk

Accepted for publication 10 April 2002

\begin{abstract}
Background/aim: The visual loss secondary to diabetic macular oedema can be controlled to some extent by photocoagulation, though the mechanism of action is largely unknown. The purpose of the present study was to quantitate the effect of photocoagulation on the blood-retinal barrier using fluorescein as a tracer of passive and active transport.

Methods: A prospective study of 46 eyes in 34 patients with clinically significant macular oedema (CSMO) examined by vitreous fluorometry before and 6 months after macular photocoagulation treatment.

Results: In 23 eyes CSMO was not present at follow up (responding eyes), in another 23 other eyes CSMO was still present (non-responding eyes). With reference to the presence or absence of CSMO at follow up, the passive transport (permeability) for responding eyes decreased after photocoagulation in contrast with an increase in non-responding eyes; the difference between the groups at follow up was significant $(p=0.03)$. The active transport for responding eyes decreased slightly at follow up, while it increased for non-responding eyes; the difference between the groups at follow up was not significant ( $\mathrm{p}=0.09$ ).

Conclusion: Following photocoagulation a reduction of diabetic macular oedema, defined as disappearance of CSMO, is paralleled by a decrease of the passive permeability while the hypothesis of an increase in the active transport from the retina to the blood could not be supported by this study.
\end{abstract}

aser treatment of clinically significant macular oedema (CSMO) in diabetes is well established and until now the -only proved treatment of CSMO as shown in the ETDRS study. ${ }^{1}$ The beneficial effect of photocoagulation is largely unknown, but it could be the result of biochemical factors or altered barrier properties of the retinal pigment epithelium such as an increase of the reabsorptive transport of fluids from the retina to the blood. ${ }^{2}{ }^{3}$

The reabsorptive, active transport has been studied with fluorescein as a tracer both in vivo and in vitro. A decrease in the transport of fluorescein from the retina to the blood shortly after photocoagulation was found in an animal study, ${ }^{4}$ probably the result of an acute inflammation as shown in other studies using histology and measurements of retinal thickness. ${ }^{56}$ After 30 days the reabsorptive transport of fluorescein increased towards the pretreatment values, but the long term effect was not examined. ${ }^{4}$ In patients with retinitis pigmentosa complicated by macular oedema the active transport of fluorescein increased in response to treatment with acetazolamide. ${ }^{7}$ Photocoagulation in patients with macular oedema might have a similar long term effect on the active transport owing to a proliferation of the retinal pigment epithelium cells causing an increase in the pumping capacity.

Another effect of photocoagulation is a decrease in passive leakage through the damaged blood-retinal barrier and a part of this could be due to endothelial repair and/or closure of leaking microaneurysms and capillaries. ${ }^{5}$ Previous studies quantitating passive and active transport have shown that the passive transport (permeability) of fluorescein through the blood-retinal barrier in increased significantly in CSMO compared both to healthy controls and to eyes with a similar degree of retinopathy without oedema. ${ }^{89}$ The active transport from the vitreous to the blood increased in diabetic retinopathy compared to healthy controls while the difference between eyes with and without oedema was small. The purpose of this study was to examine the effect of photocoagulation treatment on both types of fluorescein transport and, by inference, other electrolytes across the blood-retinal barrier.

\section{PATIENTS AND METHODS}

Patients with CSMO in at least one eye and non-proliferative retinopathy were included in the study. The patients were recruited from the outpatient clinic of the department of ophthalmology at Herlev Hospital, University of Copenhagen, and the majority of the patients were regularly followed at the Steno Diabetes Center before referral to the department.

Exclusion criteria were previous macular laser treatment, vitreous haemorrhage, and vitreous liquification as this condition does not allow calculation of blood-retinal barrier transport. ${ }^{7}$ At baseline, 48 patients (6l eyes) with CSMO were included as described earlier. ${ }^{9}$ At follow up 6 months after photocoagulation 15 eyes could not be evaluated because of to the need of peripheral photocoagulation (eight eyes), patient withdrawal (six eyes), and postponement of photocoagulation (one eye). One patient with CSMO in both eyes refused to complete the afternoon examination used for calculation of active transport at follow up and for this patient only values for passive permeability are included.

Thus, 34 patients (46 eyes) photocoagulated for CSMO were available for the study at follow up; clinical data are shown in Table 1. All participants gave their written informed consent according to the Helsinki declaration and the study was approved by the local medical committee.

\section{Methods}

Fundus photography, fluorescein angiography, vitreous fluorometry and visual acuity were performed at baseline and at follow up 6 months after photocoagulation.

CSMO was graded according to the ETDRS criteria. CSMO was defined as retinal thickening within $500 \mu \mathrm{m}$ of the foveola, hard exudates within the same $500 \mu \mathrm{m}$ if associated with retinal thickening, and $>1$ optic disc area of retinal 
Table 1 Clinical data at baseline for 34 patients with CSMO in at least one eye. $\mathrm{HbA}_{1 c}$ and blood pressure are given as cumulative values for the last year before baseline (mean of four examinations)

\begin{tabular}{llllll}
\hline & Age (years) & $\begin{array}{l}\text { Duration } \\
\text { (years) }\end{array}$ & $\mathrm{HbA}_{1 \mathrm{c}}(\%)$ & $\begin{array}{l}\text { Systolic BP } \\
(\mathrm{mm} \mathrm{Hg})\end{array}$ & $\begin{array}{l}\text { Diastolic BP } \\
(\mathrm{mm} \mathrm{Hg})\end{array}$ \\
\hline Mean & 59.38 & 11.76 & 8.57 & 143.7 & 80.89 \\
(SD) & $(7.8)$ & $(9.3)$ & $(1.6)$ & $(24.8)$ & $(8.9)$ \\
Range & $37-71$ & $1-43$ & $5-12$ & $106-195$ & $68-97$ \\
\hline
\end{tabular}

thickening if any part of the thickened retina is within 1 disc diameter from foveola. The grading was performed during biomicroscopy by an experienced retinal specialist and by a grader on stereoscopic fundus photographs. In cases of disagreement the clinical grading was given priority.

The presence or absence of CSMO at follow up was evaluated by the same experienced ophthalmologist according to the criteria mentioned above. Thus, absence of CSMO at follow up does not preclude some residual oedema.

Photocoagulation was performed shortly after the baseline examination, preferably within 1 week. The treatment strategy was to attempt to bleach prominently leaking microaneurysms (focal) and then fill with other retinal burns to cover all thickened and angiographically leaking areas (grid) with applications placed with a distance of one burn. The grid was applied to the majority of the patients (32/46-that is, 70\% received grid in addition to focal). The spot size, power, and time were 100.0 (SD 74) $\mu \mathrm{m}, 0.026(0.1) \mathrm{W}$, and $0.15(0.07)$ $\mathrm{ms}$, and the mean number of applied spots was 115 (93) using an argon green laser.

The follow up examination was made approximately 6 months after photocoagulation. In 14 eyes, more than one photocoagulation session was performed by the same experienced ophthalmologist, in case the clinical evaluation 2 months after the initial treatment indicated the need of additional laser. In three eyes, additional laser was given twice. The follow up examination was delayed in cases of additional laser, the mean time from baseline to follow up was 6.4 (SD 1.2) months.

\section{Fundus photography}

Stereoscopic colour fundus photography, 60 and 40 degrees, and fluorescein angiography were recorded using a Canon fundus camera (CF-60UV).

\section{Retinopathy grading}

Retinopathy was graded on 60 degree fundus photographs using a procedure adapted to the modified Arlie House description. ${ }^{10}$
Vitreous fluorescence was measured using an ocular fluorometer (Fluorotron, OcuMetrics, San Jose, CA, USA) from 30 minutes to 10 hours after the injection of fluorescein. The method separates fluorescein from the metabolite fluorescein glucuronide and has been described in detail earlier. ${ }^{11-13}$

Briefly, the passive transport of the blood-retinal barrier and the diffusion coefficient of fluorescein in the vitreous are calculated from the fluorescein concentration curve from 1-5 $\mathrm{mm}$ in front of the retina, obtained at 30-60 minutes after injection. With time, the flux of fluorescein from the plasma to the eye diminishes and the net movement changes towards the outward direction from the vitreous to the blood. ${ }^{14}$ The outward, active transport is calculated from the preretinal gradient and the plasma values using a computer simulation including corrections for individual differences in the diffusion coefficient for fluorescein in the vitreous and the passive permeability. ${ }^{8}$

Visual acuity was measured with standard, retroilluminated ETDRS charts.

\section{Statistics}

All calculations were performed with statistical software (Systat 7.0), the $t$ test was used to test for significance. Both eyes from the patients were included in the analyses if inclusion and exclusion criteria were fulfilled for both eyes, as macular oedema often develops in one eye months to years before the second eye. Though systemic factors probably are important for the pathophysiology, the prevalence of CSMO is also related to factors specific for each eye. Data concerning passive and active transport were log transformed before analysis to normalise distribution and all tables and figures refer to back transformed data.

\section{RESULTS}

\section{Vitreous fluorometry}

Successful treatment, defined as the absence of CSMO at follow up, was found in 23 eyes (responding eyes), while a lesser

Table 2 Passive permeability, active transport, and visual acuity in eyes with succesfull photocoagulation-that is, without CSMO at follow up ( $n=23$, responding eyes) and eyes where CSMO was still present at follow up $(n=23$, non-responding eyes). For comparison, data from eyes without CSMO both at baseline and follow up $(n=11)$ are included

\begin{tabular}{|c|c|c|c|}
\hline & $\begin{array}{l}\text { Passive permeability } \mathrm{nm} / \mathrm{s} \\
\text { baseline/follow up }\end{array}$ & $\begin{array}{l}\text { Active transport } \mathrm{nm} / \mathrm{s} \\
\text { baseline/follow up }\end{array}$ & $\begin{array}{l}\text { Visual acuity logMAR baseline/follow } \\
\text { up }\end{array}$ \\
\hline \multicolumn{4}{|c|}{ CSMO at baseline no CSMO at follow up } \\
\hline Mean & $8.23 / 7.44^{*}$ & $66.82 / 63.07$ & $0.08 / 0.05^{*}$ \\
\hline $95 \%$ confidence interval & 5.5 to $12 / 5.1$ to 11 & 42 to $107 / 43$ to 93 & 0.02 to $0.14 / 0.01$ to 0.09 \\
\hline \multicolumn{4}{|c|}{ CSMO at baseline still CSMO at follow up } \\
\hline Mean & $12.26 / 13.49$ & $67.53 / 95.15$ & $0.18 / 0.33$ \\
\hline $95 \%$ confidence interval & 8.4 to $18 / 9.5$ to 19 & 54 to $112 / 72$ to 125 & 0.07 to $0.30 / 0.21$ to 0.46 \\
\hline \multicolumn{4}{|c|}{ No CSMO at baseline and follow up } \\
\hline Mean & $3.67 \dagger / 3.95 \ddagger$ & $86.78 / 85.66$ & $0.04 / 0.04 \ddagger$ \\
\hline $95 \%$ confidence interval & 2.6 to $5.1 / 2.7$ to 5.9 & 63 to $119 / 60$ to 121 & -0.1 to $1.7 /-0.08$ to 1.7 \\
\hline
\end{tabular}

* Significant difference ( $t$ test) between responding and non-responding eyes at follow up. $†$ Significant differences ( $t$ test) between eyes without CSMO and both groups of CSMO eyes (that is, both responding and non-responding eyes). $¥$ The significant difference between eyes without CSMO and non-responding eyes with persistent CSMO at follow up. 


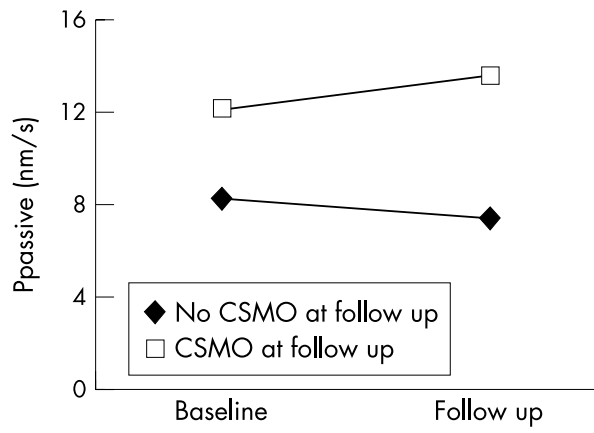

Figure 1 Passive permeability (Ppassive) of fluorescein through the blood retinal barrier at baseline and follow up for responding eyes without CSMO at follow up ( $n=23)$ compared to non-responding eyes $(n=23)$. The difference between the two groups was significant at follow up $(p=0.03)$. The mean is calculated from log transformed values.

effect of laser, defined as eyes with persistent CSMO, was found in 23 non-responding eyes.

For all 46 eyes treated, no significant differences were found for the passive or active transport from baseline to follow up (passive permeability $10.04 \mathrm{~nm} / \mathrm{s}$ and $10.01 \mathrm{~nm} / \mathrm{s}$, respectively; p $>0.1$; active transport $66.30 \mathrm{~nm} / \mathrm{s}$, and $77.84 \mathrm{~nm} / \mathrm{s}$, respectively; $\mathrm{p}>0.1$ ).

\section{Passive and active transport in relation to CSMO}

With reference to the presence of CSMO at follow up the effect of photocoagulation appeared. In responding eyes, the mean passive permeability decreased at follow up (Table 2 and Fig 1) in contrast with the increase found in non-responding eyes. Already at baseline, non-responding eyes had a lower passive permeability. However, the difference between responding and non-responding eyes was clearly non-significant at baseline ( $p$ $=0.2$, Table 2 and Fig 1 ), while at follow up the difference between the two groups was significant ( $p=0.03$ ).

The heterogeneity of the individual eyes is shown in Figure 2. For both responding and non-responding eyes the magnitude and the direction of changes from baseline to follow up varies.

The active transport was the same at baseline for responding and non-responding eyes $(\mathrm{p}=0.9$, Table 2 and Fig 3$)$. At follow up, the active transport decreased slightly in the responding eyes and increased in eyes where CSMO was still present, the difference between responding or non-responding eyes was not significant (0.09).

\section{Eyes without CSMO at baseline and follow up}

Eleven fellow eyes were available. At baseline, the passive permeability was significantly lower than for CSMO eyes and increased non-significantly with $8 \%$ at follow up (Table 2). The active transport was close to the level found in CSMO eyes and decreased slightly at follow up.

At follow up, the difference between the mean passive permeability of the eyes responding to photocoagulation compared to non-CSMO was modest (the difference between the groups was just above the level of significance; $t$ test: $\mathrm{p}=$ 0.051, Table 2).

\section{Visual acuity}

Baseline acuity was 0.082 and 0.183 in the $\log$ MAR notation for responding and non-responding eyes respectively, corresponding to a Snellen acuity of 20/25 and 20/32 (nonsignificant, $\mathrm{p}>0.1$ ). The change in visual acuity was closely related to the absence or presence of CSMO at follow up. In eyes without CSMO at follow up visual acuity increased slightly with two letters, whereas eyes with CSMO still present lost more than seven letters, the difference between groups at follow up was significant ( $p<0.001$; Table 2 ).
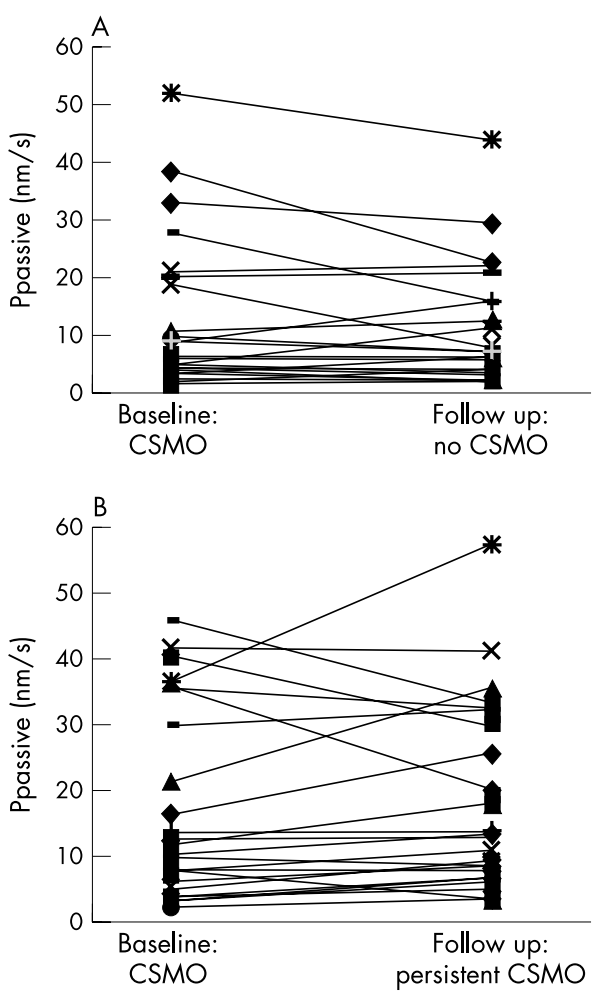

Figure 2 Passive permeability of fluorescein through the blood-retinal barrier at baseline and follow up for the individual cases both for responding $(A)$ and non-responding eyes $(B)$. The response to photocoagulation is heterogeneous corresponding to the large variance in type and extent of oedema. The mean of the same data is shown in Figure 1.

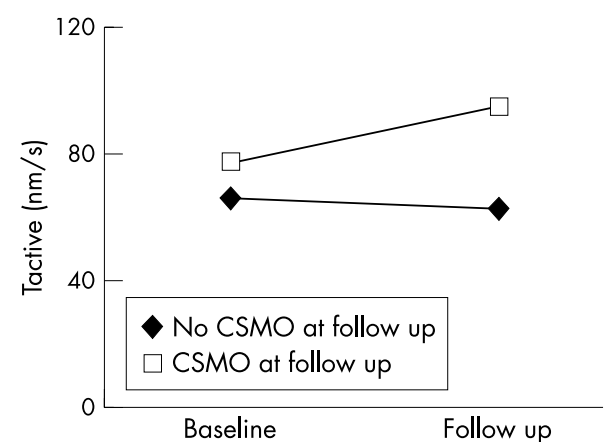

Figure 3 Active transport of fluorescein through the blood-retinal barrier at baseline and follow up for responding eyes $(n=21)$ compared to non-responding eyes $(n=23)$. The difference between the two groups was not significant at follow up, but the increase in eyes with persistent CSMO indicates that the pump mechanism is stimulated in contrast to eyes where CSMO disappeared and passive permeability decreased at follow up. The mean is calculated from log transformed values.

\section{Response in relation to oedema and retinopathy}

The area of oedema was evaluated semiquantitatively on stereo fundus photographs and tended to be lower both at baseline and at follow up in responding eyes compared to non-responding eyes $(p=0.06$ at baseline, $p=0.04$ at follow up, Mann-Whitney test). The reduction in the total oedematous area within 2 disc diameters from the fovea was $16 \%$ in responding eyes and $7 \%$ in non-responding eyes.

The effect of laser treatment was not significantly correlated with the initial type of leakage (focal, mixed, diffuse) evaluated from the fluorescein angiogram (analysis of variance). The level of retinopathy, type of photocoagulation 
(focal/grid), number of photocoagulation spots and energy delivered were also insignificant for the outcome.

\section{DISCUSSION}

The primary effect of laser treatment seems to be the result of a reduction in the passive leakage and the present study does not support the hypothesis that photocoagulation increases the active, reabsorbing transport from the retina to the blood.

For ethical reasons, no control group was included and the modest reduction in passive permeability in eyes without CSMO at follow up has to be compared with eyes with persistent CSMO where an increase was seen and, probably, untreated eyes will increase substantially in passive permeability. Also, laser treatment is given only sectorially in the region of retinal thickening, and even though vitreous fluorometry is highly correlated to the fluorescein leakage in the posterior pole the full effect might not be detected as vitreous fluorometry is based on measurements of the fluorescein concentration along the optical axis of the eye. It is interesting, however, that the passive permeability of the responding eyes at follow up was rather close to eyes without CSMO and as a part of the difference might be due to a difference in retinopathy, ${ }^{9}$ it is possible that the modest reduction in passive permeability in responding eyes is sufficient to cross a threshold value.

The mechanisms underlying the decrease in passive transport cannot be deduced from the present study. Stefansson et al have shown that the oxygen tension in the preretinal vitreous increases after photocoagulation, owing to increased transport through the retinal pigment epithelium at the laser spots and/or decrease in oxygen consumption as the number of photoreceptors are reduced. ${ }^{15}$ The improved oxygenation constricts the retinal arterioles due to autoregulation and thus a decrease in capillary and venous hydrostatic pressure ${ }^{16-21}$ and, in theory, the passive permeability should decrease. In the present study no significant correlation was found regarding the number of laser spots and the change in passive permeability.

At baseline, the passive permeability was lower in responding eyes compared to non-responding eyes. However, the difference at baseline was not significant and no other parameters at baseline or photocoagulation strategy factors seemed significant for the outcome. This could be due to the limited size of the study and the heterogeneity of the patients.

The reabsorptive, active transport did not increase in eyes responding to photocoagulation. Rather, the active transport seems to change in the same direction as the passive transport, indicating that photocoagulation by itself does not stimulate the active transport. Most likely, the level of electrolytes in the retina might be a stimulus for the pump mechanism as the active transport increased significantly from baseline to follow up (from 67 to $95 \mathrm{~nm} / \mathrm{s}, \mathrm{p}=0.02$ ) in eyes with persistent CSMO.

For all eyes included (both responding and nonresponding), the visual acuity improved by more than one line in $15 \%$ and decreased by more than one line in $30 \%$, corresponding to earlier studies with an overall stabilisation of visual acuity. ${ }^{11-23}$

\section{CONCLUSION}

Clinically significant macular oedema disappeared after laser treatment in $50 \%$ of the eyes and the passive permeability of fluorescein followed the clinical course and visual acuity. The relation between changes in the transport of fluorescein and the retinal movements of electrolytes and water is unknown; however, the modest reduction of passive transport found in this study was sufficient to remove CSMO and the passive transport at follow up was closed to eyes without CSMO. In contrast, the active reabsorptive transport seemed to be unrelated to the beneficial effect of photocoagulation, as it was stable in eyes where CSMO disappeared and increased in eyes with persistent oedema.

\section{ACKNOWLEDGEMENT}

The study was funded by The Danish Diabetes Association, Odense and Danish Eye Health Society, Copenhagen.

\section{Authors' affiliations}

B Sander, M Larsen, C Engler, B Moldow, H Lund-Andersen

Department of Ophthalmology, Herlev Hospital, University of

Copenhagen, Dk 2730 Herlev, Denmark

\section{REFERENCES}

1 ETDRS report number 1. Photocoagulation for diabetic macular edema. Arch Ophthalmol 1985;103:1796-806

2 Guyot-Argenton C, Bernard J, Favard G, et al. Grid photocoagulation for focal diabetic macular edema with focal leaks and hard-exudates involving the perifoveal area. Doc Ophthalmol 1999;97:412-25.

3 Wallow I. Repair of the pigment epithelium barrier following photocoagulation. Arch Ophthalmol 1984;102:126-35.

4 Zweig K, Cunha-Vaz J, Peyman G, et al. Effect of argon laser photocoagulation on fluorescein transport across the blood-retina barrier Exp Eye Res 1981;32:323-9.

5 Wilson D, Finkelstein D, Quigley H, et al. Macular grid photocoagulation. An experimental study on the primate retina. Arch Ophthalmol 1988;106:100-5.

6 Tsujikawa A, Kiryu J, Dong J, et al. Quantitative analysis of diabetic macular edema after scatter phtocoagulation with the scanning retinal thickness analyzer. Retina 1999;19:59-64.

7 Moldow B, Sander B, Larsen M, Engler C, et al. The effect of acetazolamide on passive and active transport of fluorescein across the blod-retina barrier in retinitis pigmentosa complicated by macular edema. Graefes Arch Clin Exp Ophthalmol 1998;236:881-9.

8 Sander B, Larsen M, Moldow B, et al. Passive and active transport of fluorescein in diabetic macular edema. Introducing a simplified mathematical model of active transport. Invest Ophthalmol Vis Sci 2001:42:433-8.

9 Sander B, Larsen M, Engler C. Diabetic macular oedema: A comparison between vitreous fluorometry, angiography and retinopathy. $\mathrm{Br} J$ Ophthalmol 2002;86:316-20.

10 ETDRS report number 10 . Grading diabetic retinopathy from stereoscopic color fundus photographs-an extension of the modified Arlie House classification. Ophthalmology 1991;98:786-807.

11 Larsen M, Dalgaard P, Lund-Andersen H. Differential spectrofluoremetry in the human vitreous: blood-retina barrier permeability to fluorescein and fluorescein glucuronide. Graefes Arch Clin Exp Ophthalmol 1991;229:350-7.

12 Lund-Andersen $\mathbf{H}$, Krogsaa B, La Cour $M$, et al. Quantitative vitreous fluorophotometry applying a mathematical model of the eye. Invest Ophthalmol Vis Sci 1985;26:698-710.

13 Dalgaard $\mathbf{P}$, Larsen $M$. Fitting numerical solutions of differential equations to experimental data: a case study and some general remarks. Biometrics 1990;46:1097-109.

14 Engler C, Sander B, Larsen M, et al. Fluorescein transport across the human blod-retina barrier in the direction vitreous to blood. Acta Ophthalmol 1994;72:655-62.

15 Stefánsson $\mathbf{E}$. The therapeutic effects of retinal laser treatment and vitrectomy. A theory based on oxygen and vascular physiology. Acta Ophthalmol 2001;79:435-40.

16 Stefánsson E, Landers $M$, Wolbarsht $M$. Oxygenation and vasodilation in relation to diabetic and other proliferative retinopathies. Ophthalmic Surg 1983;14:209-26.

17 Kristinsson J, Gottfredsdóttir M, Stefánsson E, et al. Retinal vessel dilation and elongation in diabetic macular oedema. $\mathrm{Br} J$ Ophthalmol 1997;81:274-8.

18 Gottfredsdóttir $M$, Stefánsson E, Gíslason I. Retinal vasoconstriction after laser treatment for diabetic macular edema. Am J Ophthalmol 1993; 1 15:64-7.

19 Rassam S, Patel V, Kohner E. The effect of experimental hypertension on retinal vascular autoregulation in humans: a mechanism for the progression of diabetic retinopathy. Exp Physiol 1995:80:53-60.

20 Patel V, Rassam S, Newsom R, et al. Retinal blood flow in diabetic retinopathy. BN 1992;305:678-83.

21 Ladas I, Theodossiadis. Long-term effectiveness of modified grid laser photocoaulation for diffuse macular edema. Acta Ophthalmol 1993:71:393-7.

22 Lee CM, Olk RJ. Modified grid laser photocoagulation for diffuse macular oedema, long-term visual results. Ophthalmology 1991;98:1594-602.

23 Bek T, Møller F, Klausen B. Short term visual prognosis after retinal laser photocoagulation for diabetic maculopathy. Acta Ophthalmol 2000;78:539-42. 